\title{
PRESCRIBING THE BINARY DIGITS OF PRIMES
}

\author{
JEAN BOURGAIN
}

\begin{abstract}
We present a new result on counting primes $p<N=2^{n}$ for which $r$ (arbitrarily placed) digits in the binary expansion of $p$ are specified. Compared with earlier work of Harman and Katai, the restriction on $r$ is relaxed to $r<c\left(\frac{n}{\log n}\right)^{4 / 7}$. This condition results from the estimates of Gallagher and Iwaniec on zero-free regions of $L$-functions with 'powerful' conductor.
\end{abstract}

\section{(0). Summary}

This work is motivated by the paper $[\mathrm{H}-\mathrm{K}]$ on the problem described in the title. We prove the following

Theorem. Let $N=2^{n}$, $n$ large enough, and $A \subset\{1, \ldots, n-1\}$ such that

$$
r=|A|<c\left(\frac{n}{\log n}\right)^{4 / 7}
$$

Then, considering binary expansions $x=\sum_{j<n} x_{j} 2^{j}\left(x_{0}=1\right.$ and $x_{j}=0,1$ for $1 \leq j<n)$ and assignments $\alpha_{j}$ for $j \in A$, we have

$$
\mid\left\{p<N ; \text { for } j \in A \text {, the } j \text {-digit of } p \text { equals } x_{j}\right\} \mid \sim 2^{-r} \frac{N}{\log N} \text {. }
$$

A few comments. Statement (0.2) can also be formulated as an asymptotic formula. Next, our result has an analogue for $q$-ary expansions (with essentially the same proof) and we restricted ourselves to $q=2$ only for simplicity.

This research was partially supported by NSF grants DMS-0808042 and DMS-0835373. 
The paper $[\mathrm{H}-\mathrm{K}]$ establishes a similar result under the assumption

$$
|A|<c \frac{\sqrt{n}}{\log n}
$$

A key ingredient in $[\mathrm{H}-\mathrm{K}]$ and also here is the use of zero-free regions for $L$-functions $L(s, \chi)$. In particular, the restriction (0.1) follows from results of Gallagher and Iwaniec ([G], [I]) that provide the zero-free region $1-\sigma<$ $c \frac{1}{(\log q T \cdot \log \log q T)^{3 / 4}},|\gamma|<T$ where $\rho=\sigma+i \gamma$, for special moduli $q$ that are powers of a fixed integer (here $q=2^{j}$ ). This region is larger than what's available in the general case. Note that we dismiss here a possible Siegel zero, which is not a concern for $q$ specified as above. Although the Gallagher-Iwaniec theorem is one of the elements in the $[\mathrm{H}-\mathrm{K}]$ argument, its full potential was unfortunately not exploited. As in $[\mathrm{H}-\mathrm{K}]$, we use the circle method but with a smaller minor arcs region, leading to more saving for that contribution. As one expects, the major arcs analysis is more involved.

Note that there are two multiplicative character sums entering the discussion (after conversion of the exponential sums in the circle method)

$$
\sum_{n<N} \Lambda(n) \chi(n)
$$

and

$$
\sum_{n<N} f(n) \chi(n)
$$

where $f=1_{\left[x_{j}=\alpha_{j}\right.}$ for $\left.j \in A\right]$ or a suitably modified version.

The main additional idea in this paper may be roughly explained as follows. In general, again dismissing Siegel zeros, $L(s, \chi)$ has a zero-free region $1-\sigma<\frac{c}{\log q T}$, $|\gamma|<T$. But, as implied by the usual density estimates, for 'most' characters, this zero-free region is much larger leading to better bounds on (0.4). For the remaining 'bad' characters, which are few (including possible Siegel zeros), we seek non-trivial estimates on the sum (0.5). This is clearly reasonable, in view of the additive structure of $f$. Perhaps not surprisingly, it turns out that the only situation that escapes this analysis (that in fact can be carried out as long 
$r<n^{\frac{2}{3}-\varepsilon}$ ) are precisely moduli of the form $2^{j}$. Thus at the end, it is the size of the $[\mathrm{G}],[\mathrm{I}]$ zero-free region that dictates the restriction on $|A|$.

\section{Minor Arcs Contribution}

Let $N=2^{n}$. Write

$$
\sum_{k \leq N} \Lambda(k) f(k)=\int_{0}^{1} S(\alpha) \bar{S}_{f}(\alpha) d \alpha
$$

denoting

$$
S(\alpha)=\sum \Lambda(k) e(k \alpha)
$$

and

$$
S_{f}(\alpha)=\sum f(k) e(k \alpha) .
$$

We assume $f(k)=0$ for $k$ even, since obviously $k \equiv 1(\bmod 2)$ is a necessary condition.

We fix a parameter $B=B(n)$ which will be specified in $\S 6$. At this point, let us just say that $\log B \sim n^{4 / 7}$, up to logarithmic factors.

The major arcs are defined by

$$
\mathcal{M}(q, a)=\left[\left|\alpha-\frac{a}{q}\right|<\frac{B}{q N}\right] \text { where } q<B
$$

Given $\alpha$, there is $q<\frac{N}{B}$ such that

$$
\left|\alpha-\frac{a}{q}\right|<\frac{B}{q N}<\frac{1}{q^{2}}
$$

From Vinogradov's estimate (Theorem 13.6 in [I-K])

$$
\begin{aligned}
|S(\alpha)| & <\left(q^{\frac{1}{2}} N^{\frac{1}{2}}+q^{-\frac{1}{2}} N+N^{\frac{4}{5}}\right)(\log N)^{3} \\
& \ll\left(\frac{N}{\sqrt{B}}+\frac{N}{\sqrt{q}}+N^{4 / 5}\right)(\log N)^{3} .
\end{aligned}
$$

Hence if $q \geq B$,

$$
|S(\alpha)| \ll \frac{N}{\sqrt{B}}(\log N)^{3} .
$$


Thus the minor arcs contribution in (1.1) is at most

$$
\ll \frac{N}{\sqrt{B}}(\log N)^{3}\left\|S_{f}\right\|_{1} .
$$

Write $k=\sum_{0 \leq j<n} k_{j} 2^{j}$ with $k_{j}=0,1$. Given $A \subset\{1, \ldots, n-1\}$ and $\alpha_{j}=0$ or 1 for $j \in A$, we define

$$
f(x)=\prod_{j \in A} h_{\alpha_{j}}\left(\frac{x}{2^{j+1}}\right)
$$

where $0 \leq h_{0} \leq 1$ is a smooth 1-periodic function satisfying

$$
\begin{cases}h_{0}(t)=1 & \text { if } \frac{1}{n} \leq t \leq \frac{1}{2}-\frac{1}{n} \\ h_{0}(t)=0 & \text { if } \frac{1}{2} \leq t \leq 1\end{cases}
$$

( $h_{1}$ is defined similarly). Thus

$$
\left\|\hat{h}_{\alpha}\right\|_{1}<C \log n
$$

and, by approximation, we can assume that say

$$
\operatorname{supp} \hat{h}_{\alpha} \subset\left[-n^{3}, n^{3}\right]
$$

the additional error term (for suitably chosen $h_{\alpha}$ ) being at most $e^{-n}<\frac{1}{N}$ and hence may be ignored.

Note that in the formulation of the main theorem, we did not specify the error term as we do not intend to put emphasis on this aspect. Thus the function $f$ introduced in (1.8) suffices for our purpose, while establishing an asymptotic formula with specified error term would require an approximation of the functions $1_{\left[0, \frac{1}{2}[\right.}$ and $1_{\left[\frac{1}{2}, 1[\right.}$ which is better than given by $h_{0}$ and $h_{1}$.

In particular $f$ satisfies

$$
\left\|S_{f}\right\|_{1}<(C \log n)^{|A|} .
$$

Substitution in (1.7) gives then the bound

$$
\frac{N}{\sqrt{B}}(C \log n)^{|A|} n^{3} .
$$




\section{Major Arcs Analysis (I)}

Next, we analyze the major arcs contributions $(q<B)$

$$
\sum_{(a, q)=1} \int_{\left|\alpha-\frac{a}{q}\right|<\frac{B}{q N}} S(\alpha) \bar{S}_{f}(\alpha) d \alpha
$$

Write $\alpha=\frac{a}{q}+\beta$. Defining

$$
\tau(\chi)=\sum_{m=1}^{q} \chi(m) e_{q}(m)
$$

we have (see [D], p. 147)

$$
S(\alpha)=\frac{1}{\phi(q)} \sum_{\chi} \tau(\bar{\chi}) \chi(a)\left[\sum_{k \leq N} \chi(k) \Lambda(k) e(k \beta)\right]+O\left((\log N)^{2}\right)
$$

where by partial summation

$$
\begin{aligned}
\left|\sum_{k \leq N} \chi(k) \Lambda(k) e(k \beta)\right| & \leq(1+N|\beta|) \max _{u<N}|\psi(u, \chi)| \\
& <\frac{B}{q} \max _{u<N}|\psi(u, \chi)| .
\end{aligned}
$$

We subdivide the multiplicative characters $\chi$ in classes $\mathcal{G}$ and $\mathcal{B}$ (to be specified), depending on the zero set of $L(s, \chi)$.

Thus

$$
S(\alpha)=\frac{\mu(q)}{\phi(q)}\left[\sum_{k \leq N} \Lambda(k) e(k \beta)\right]+(2.4)+(2.5)
$$

where the first term comes from the contribution of the principal character $\chi_{0}$ and

$$
(2.4)=\frac{1}{\phi(q)} \sum_{\substack{\chi(\bmod q) \\ \chi \in \mathcal{G}}} \tau(\bar{\chi}) \chi(a)\left[\sum_{k \leq N} \chi(k) \Lambda(k) e(k \beta)\right]
$$

and

$$
(2.5)=\frac{1}{\phi(q)} \sum_{\substack{\chi(\bmod q) \\ \chi \in \mathcal{B}}} \cdots
$$


Hence

$$
|(2.4)| \lesssim \frac{B}{\sqrt{q}}\left[\max _{\substack{u<N \\ \chi(\bmod q), \chi \in \mathcal{G}}}|\psi(u, \chi)|\right]
$$

by (2.3). The contribution of (2.6) in (2.1) is at most

$$
\int_{\bigcup_{(a, q)=1} \mathcal{M}(q, a)}\left|S_{f}(\alpha)\right| d \alpha .
$$

Summing over $q<B$ and using the bound (1.10) gives the estimate

$$
B(C \log n)^{|A|}\left\{\max _{\substack{u<N \\ \chi \in \mathcal{G}}}|\psi(u, \chi)|\right\}
$$

where in (2.7) the conductor of $\chi$ is at most $B$.

\section{Major Arcs Analysis (II)}

Next we treat $\chi=\chi_{0}$ and $\chi \in \mathcal{B}$.

Assume $\chi$ is induced by $\chi_{1}$ which is primitive $\left(\bmod q_{1}\right), q_{1} \mid q$. Then from $[\mathrm{D}]$, p. 67

$$
\tau(\bar{\chi})=\mu\left(\frac{q}{q_{1}}\right) \bar{\chi}_{1}\left(\frac{q}{q_{1}}\right) \tau\left(\bar{\chi}_{1}\right)
$$

which vanishes, unless $q_{2}=\frac{q}{q_{1}}$ is square free with $\left(q_{1}, q_{2}\right)=1$.

The contribution of $\chi$ in (2.1) equals

$$
\frac{\tau(\bar{\chi})}{\phi(q)} \int_{|\beta|<\frac{B}{q N}}\left[\sum_{k \leq N} \chi(k) \Lambda(k) e(k \beta)\right]\left[\sum_{k<N} f(k)\left(\sum_{a=1}^{q} \chi(a) e_{q}(-a k)\right) e(-k \beta)\right] d \beta .
$$

We have

$$
\begin{aligned}
\sum_{a=1}^{q} e_{q}(a k) \chi(a) & =\sum_{(a, q)=1} e_{q}(a k) \chi_{1}(a) \\
& =\left[\sum_{\left(a_{1}, q_{1}\right)=1} e_{q_{1}}\left(a_{1} k\right) \chi_{1}\left(a_{1}\right)\right]\left[\sum_{\left(a_{2}, q_{2}\right)=1} e_{q_{2}}\left(a_{2} k\right)\right]
\end{aligned}
$$




$$
=\chi_{1}(k) \tau\left(\chi_{1}\right) c_{q_{2}}(k)
$$

(cf. [D], p. 65) and

$$
c_{q_{2}}(k)=\frac{\mu\left(\frac{q_{2}}{\left(q_{2}, k\right)}\right) \phi\left(q_{2}\right)}{\phi\left(\frac{q_{2}}{\left(q_{2}, k\right)}\right)} .
$$

(cf. [D], p. 149).

From (3.1), (3.3), (3.4)

$$
\begin{aligned}
\frac{\tau(\bar{\chi})}{\phi(q)}\left[\sum_{a=1}^{q} \chi(a) e_{q}(-a k)\right] & =\frac{\left|\tau\left(\chi_{1}\right)\right|^{2}}{\phi\left(q_{1}\right)} \frac{\bar{\chi}_{1}\left(q_{2}\right)}{\phi\left(\frac{q_{2}}{\left(q_{2}, k\right)}\right)} \mu\left(\left(q_{2}, k\right)\right) \chi_{1}(k) \\
& =\frac{q_{1}}{\phi\left(q_{1}\right)} \frac{\bar{\chi}_{1}\left(q_{2}\right)}{\phi\left(\frac{q_{2}}{\left(q_{2}, k\right)}\right)} \mu\left(\left(q_{2}, k\right)\right) \chi_{1}(k)
\end{aligned}
$$

(cf. [D], p. 66).

Returning to (3.2), rather than integrating in $\beta$ over the interval $|\beta|<\frac{B}{q N}$, we introduce a weight function

$$
w\left(\frac{q N}{B} \beta\right)
$$

where $0 \leq w \leq 1$ is a smooth bumpfunction on $\mathbb{R}$ such that $w=1$ on $[-1,1]$, supp $w \subset[-2,2]$ and

$$
|\hat{w}(y)|<C e^{-|y|^{1 / 2}} .
$$

(Note that this operation creates in (2.1) an error term that is captured by the minor arcs contribution (1.7)).

Hence, substituting (3.5), (3.2) becomes

$$
\frac{q_{1}}{\phi\left(q_{1}\right)} \overline{\mathcal{X}}_{1}\left(q_{2}\right) \frac{B}{q N} \sum_{k_{1}, k_{2}<N} \hat{w}\left(\frac{B}{q N}\left(k_{1}-k_{2}\right)\right) \mathcal{X}\left(k_{1}\right) \Lambda\left(k_{1}\right) f\left(k_{2}\right) \frac{u\left(\left(q_{2}, k_{2}\right)\right)}{\phi\left(\frac{q_{2}}{\left(q_{2}, k_{2}\right)}\right)} \mathcal{X}_{1}\left(k_{2}\right)
$$

and we observe that by our assumption on $\hat{w}$ the $k_{1}, k_{2}$ summation in (3.6) is restricted to $\left|k_{1}-k_{2}\right|<\frac{q N}{B} n^{3}$, up to a negligible error.

We first examine the contribution of the principal characters. 
For $\mathcal{X}=\mathcal{X}_{0}, q_{1}=1$ and (3.6) becomes

$$
\frac{B}{q N} \sum_{k_{1}, k_{2}<N} \hat{w}\left(\frac{B}{q N}\left(k_{1}-k_{2}\right)\right) \Lambda\left(k_{1}\right) f\left(k_{2}\right) \frac{\mu\left(\left(q, k_{2}\right)\right)}{\phi\left(\frac{q}{\left(q, k_{2}\right)}\right)} .
$$

Recall the distributional property of the primes ([I-K], Corollary 8.30 )

$$
\sum_{k \leq x} \Lambda(k)=x+O\left(x \exp \left(-c(\log x)^{3 / 5}(\log \log x)^{-\frac{1}{5}}\right)\right)
$$

and also our assumption $\log B \ll n^{4 / 7}$. Performing the $k_{1}$-summation in (3.7), partial summation together with (3.8) give

$$
\begin{aligned}
& \frac{B}{q N} \sum_{k_{1}, k_{2}} \hat{w}\left(\frac{B}{q N}\left(k_{1}-k_{2}\right)\right) f\left(k_{2}\right) \frac{\mu\left(\left(q, k_{2}\right)\right)}{\phi\left(\frac{q}{\left(q, k_{2}\right)}\right)}+O\left(N \exp \left(-c(\log N)^{3 / 5}(\log \log N)^{-\frac{1}{5}}\right)\right) \\
& =\sum_{k} f(k) \frac{\mu((q, k))}{\phi\left(\frac{q}{(q, k)}\right)}+O\left(N \exp \left(-c(\log N)^{3 / 5}(\log \log N)^{-\frac{1}{5}}\right)\right)
\end{aligned}
$$

Let $\kappa(q)$ be a function satisfying the following

Assumption A. Let $q_{0}<B$ be odd and square free. Then

$$
\sum_{q_{0} \mid k, k<N} f(k)=\mathbb{E}[f] \frac{N}{q_{0}}+O\left(\kappa\left(q_{0}\right) \frac{N}{q_{0}} 2^{-r}\right)
$$

where $\mathbb{E}[f]$ denotes the normalized average.

Remark. Since the function $\kappa(q)$ at this stage remains unspecified and its properties will be established later (in $\S 4$ ), we thought this presentation more desirable than making a more technical statement here (cf. (4.26)). 
Assuming $q$ square-free (sf) and odd, the first term of (3.9) equals

$$
\begin{aligned}
& \sum_{q^{\prime} \mid q} \frac{\mu\left(q^{\prime}\right)}{\phi\left(\frac{q}{q^{\prime}}\right)}\left[\sum_{(q, k)=q^{\prime}} f(k)\right]= \\
& \sum_{q^{\prime} \mid q} \frac{\phi\left(q^{\prime}\right)}{\phi(q)} \mu\left(q^{\prime}\right) \sum_{q^{\prime \prime} \mid \frac{q}{q^{\prime}}} \mu\left(q^{\prime \prime}\right)\left[\sum_{\substack{q^{\prime} q^{\prime \prime} \mid k \\
k<N}} f(k)\right]
\end{aligned}
$$

and substituting (3.10) we obtain

$$
\begin{gathered}
N \mathbb{E}[f] \sum_{q^{\prime} \mid q} \frac{\mu\left(q^{\prime}\right)}{\phi\left(q / q^{\prime}\right)} \sum_{q^{\prime \prime} \mid \frac{q}{q^{\prime}}} \frac{\mu\left(q^{\prime \prime}\right)}{q^{\prime} q^{\prime \prime}} \\
+O\left(N 2^{-r} \sum_{q^{\prime} \mid q} \frac{\phi\left(q^{\prime}\right)}{\phi(q)} \sum_{q^{\prime \prime} \mid \frac{q}{q^{\prime}}} \frac{\kappa\left(q^{\prime} q^{\prime \prime}\right)}{q^{\prime} q^{\prime \prime}}\right) .
\end{gathered}
$$

Next

$$
\begin{aligned}
(3.11) & =N \mathbb{E}[f] \sum_{q^{\prime} \mid q} \frac{\mu\left(q^{\prime}\right)}{\phi\left(q / q^{\prime}\right)} \frac{1}{q^{\prime}} \prod_{p \mid \frac{q}{q^{\prime}}}\left(1-\frac{1}{p}\right) \\
& =N \mathbb{E}[f] \sum_{q^{\prime} \mid q} \frac{\mu\left(q^{\prime}\right)}{\phi\left(q / q^{\prime}\right)} \frac{1}{q} \phi\left(q / q^{\prime}\right) \\
& = \begin{cases}N \mathbb{E}[f] & \text { if } q=1 \\
0 & \text { otherwise. }\end{cases}
\end{aligned}
$$

Summing (3.12) over $q<B$ sf and odd, we have the estimate (setting $q_{1}=$ $\left.q^{\prime} q^{\prime \prime}\right)$

$$
2^{-r} N \sum_{\substack{q_{1}<B \\ q_{1} s f}} \frac{\kappa\left(q_{1}\right)}{q_{1}}\left[\sum_{\substack{q^{\prime \prime} \mid q_{1},\left(q_{1}, q_{2}\right)=1 \\ q_{2}<B s f}} \frac{1}{\phi\left(q^{\prime \prime}\right) \phi\left(q_{2}\right)}\right]
$$




$$
<2^{-r} N(\log B)^{2}\left[\sum_{\substack{q<B \\ q s f, \text { odd }}} \frac{\kappa(q)}{q}\right] .
$$

For $q$ sf and even, set $q=2 q_{1}$ and note that the first term of (3.9) equals

$$
\sum_{q^{\prime} \mid q_{1}} \frac{\mu\left(q^{\prime}\right)}{\phi\left(q_{1} / q^{\prime}\right)}\left[\sum_{\left(q_{1}, k\right)=q^{\prime}} f(k)\right]
$$

and we proceed similarly as above with the same conclusion and $q$ replaced by $q_{1}$. From the preceding, the contribution of the principal characters equals

$$
2 \mathbb{E}[f] N+C N 2^{-r}(\log N)^{2}\left[\sum_{\substack{q<B \\ q s f}} \frac{\kappa(q)}{q}\right]+B N \exp \left(-c(\log N)^{3 / 5}(\log \log N)^{-\frac{1}{5}}\right) .
$$

Consider next $\chi_{1} \in \mathcal{B}, \chi_{1}$ primitive.

Returning to (3.6), estimate by

$$
\begin{aligned}
& \quad \frac{q_{1}}{\phi\left(q_{1}\right)} \frac{B}{q N} \sum_{k_{1}<N}\left|\sum_{k} \hat{w}\left(\frac{B}{q N}\left(k_{1}-k\right)\right) f(k) \frac{\mu\left(\left(q_{2}, k\right)\right)}{\phi\left(\frac{q_{2}}{\left(q_{2}, k\right)}\right)} \mathcal{X}_{2}(k)\right| \\
& \leq \frac{q_{1}}{\phi\left(q_{1}\right)} \frac{B}{q} \sum_{q_{2}^{\prime} \mid q_{2}} \frac{\phi\left(q_{2}^{\prime}\right)}{\phi\left(q_{2}\right)} \max _{k_{1}}\left|\sum_{k,\left(k, q_{2}\right)=q_{2}^{\prime}} \hat{w}\left(\frac{B}{q_{N}}\left(k_{1}-k\right)\right) f(k) \mathcal{X}_{1}(k)\right|
\end{aligned}
$$

We introduce another function $\alpha\left(q_{1}, q_{0}\right)$ satisfying

\section{Assumption B.}

Given $q_{0}, q_{1}<B,\left(q_{0}, q_{1}\right)=1$ with $q_{0} s f$ and odd, $\chi_{1}\left(\bmod q_{1}\right)$ primitive,

$$
\left|\sum_{k \in J, q_{0} \mid k} f(k) \chi_{1}(k)\right|<2^{-r} \alpha\left(q_{1}, q_{0}\right) \frac{|J|}{q_{0}}
$$

holds, whenever $J \subset[1, N]$ is an interval of size $\sim \frac{N}{B}$. 
The function $\alpha\left(q_{1}, q_{0}\right)$ which enters in $(3.17)$ will be studied in $\S 4, \S 5$ and in particular can be chosen to satisfy (5.11). Partial summation using the decay estimate on $\hat{w}$ and (3.17) implies the following bound on (3.16)

$$
2^{-r} N(\log N)^{3} \frac{q_{1}}{\phi\left(q_{1}\right)} \sum_{q_{2}^{\prime} \mid q_{2}} \frac{\phi\left(q_{2}^{\prime}\right)}{\phi\left(q_{2}\right)} \sum_{q_{2}^{\prime \prime} \mid \frac{q_{2}}{q_{2}^{\prime}}} \frac{\alpha\left(q_{1}, q_{2}^{\prime} q_{2}^{\prime \prime}\right)}{q_{2}^{\prime} q_{2}^{\prime \prime}}
$$

Summation of (3.18) over sf $q_{2}<B,\left(q_{1}, q_{2}\right)=1$ gives the following bound for the $\mathcal{X}_{1}$-contribution

$$
\begin{gathered}
2^{-r} N(\log N)^{3} \frac{q_{1}}{\phi\left(q_{1}\right)} \sum_{\substack{q_{3}<B \\
s f, \text { odd }}} \frac{\alpha\left(q_{1}, q_{3}\right)}{q_{3}} \sum_{\substack{q_{2}^{\prime \prime} \mid q_{3} \\
q_{2}^{\prime \prime \prime}<B,\left(q_{2}^{\prime \prime}, q_{2}^{\prime \prime}\right)=1}} \frac{1}{\phi\left(q_{2}^{\prime \prime}\right) \phi\left(q_{2}^{\prime \prime \prime}\right)} \\
<2^{-r} N(\log N)^{5} \frac{q_{1}}{\phi\left(q_{1}\right)}\left\{\sum_{\substack{q_{3}<B \\
s f \text { odd }}} \frac{\alpha\left(q_{1}, q_{3}\right)}{q_{3}}\right\} .
\end{gathered}
$$

The necessary information on the functions $\kappa(q)$ and $\alpha\left(q_{1}, q_{0}\right)$ needed to bound (3.15) and (3.19) will be established in the next sections $\S 4, \S 5$.

\section{Further Estimates (I)}

Consider condition (3.10) and the expression (3.14).

Let

$$
A=\left\{j_{1}<j_{2}<\cdots<j_{r}\right\} \subset\{1, \ldots, n\} .
$$

Let $1<J_{1}<J_{2}<n$ (to be specified) and write

$$
x=\sum_{j<J_{1}} x_{j} 2^{j}+\sum_{J_{1} \leq j<J_{2}} x_{j} 2^{j}+\sum_{J_{2} \leq j<n} x_{j} 2^{j}=u+v+w .
$$

We evaluate (assuming $q_{0}$ odd)

$$
\sum_{q_{0} \mid x} f(x)=\sum_{u, w}\left[\sum_{q_{0} \mid u+v+w} f(u+v+w)\right]
$$

by evaluating the inner sum in $v$ with $u, w$ fixed. 
Clearly

$$
\begin{gathered}
f(x)=\prod_{\substack{j \in A \\
2^{j+1}}} h\left(\frac{x}{2^{j \in A}}\right)= \\
\prod_{j \leq J_{1}} h\left(\frac{u}{2^{j+1}}\right) \cdot \prod_{\substack{j \in A \\
J_{1}<j \leq J_{2}}}\left[h\left(\frac{v}{2^{j+1}}\right)+O\left(\|h\|_{C^{1}} 2^{J_{1}-j}\right)\right] \cdot \prod_{\substack{j \in A \\
J_{2}<j \leq n}}\left[h\left(\frac{w}{2^{j+1}}\right)+O\left(\|h\|_{C^{1}} 2^{J_{2}-j}\right)\right] .
\end{gathered}
$$

Fix $\Delta J=J_{2}-J_{1}>10 \log B$ and take $J_{1}, J_{2}$ satisfying

$$
\operatorname{dist}\left(\left\{J_{1}, J_{2}\right\}, A\right)>\frac{n}{10 r}
$$

and

$$
\left|A \cap\left[J_{1}, J_{2}\right]\right|<\frac{10 r \Delta J}{n} .
$$

Since $\|h\|_{C^{1}} \lesssim n^{2}$, assuming

$$
r<10^{-6} \frac{n}{\log n}
$$

we have

$$
f_{-} \leq f \leq f_{+}
$$

with

$$
f_{ \pm}(x)=\prod_{\substack{j \in A \\ j \leq J_{1}}} h\left(\frac{u}{2^{j+1}}\right) \cdot \prod_{\substack{j \in A \\ J_{1}<j \leq J_{2}}} h_{ \pm}\left(\frac{v}{2^{j+1}}\right) \cdot \prod_{\substack{j \in A \\ j>J_{2}}} h_{ \pm}\left(\frac{w}{2^{j+1}}\right)
$$

and

$$
h_{ \pm}=h \pm 2^{-\frac{n}{11 r}}
$$

Note that

$$
\mathbb{E}\left[f_{+}\right]-\mathbb{E}\left[f_{-}\right] \lesssim r 2^{-\frac{n}{11 r}} 2^{-r}<2^{-\frac{n}{12 r}} 2^{-r}
$$

Obviously

$$
\sum_{q_{0} \mid x} f_{-}(x) \leq \sum_{q_{0} \mid x} f(x) \leq \sum_{q_{0} \mid x} f_{+}(x)
$$

and hence

$$
\left|\sum_{q_{0} \mid x} f(x)-\frac{N}{q_{0}} \mathbb{E}[f]\right|<\max _{ \pm}\left|\sum_{q_{0} \mid x} f_{ \pm}(x)-\frac{N}{q_{0}} \mathbb{E}\left[f_{ \pm}\right]\right|+2^{-\frac{n}{12 r}} \frac{N}{q_{0}} 2^{-r}
$$


Assume that for fixed $u, w$

$$
\begin{gathered}
\left|\sum_{\substack{v \equiv-u-w \\
\left(\bmod q_{0}\right)}} \prod_{\substack{j \in A \\
J_{1}<j \leq J_{2}}} h_{+}\left(\frac{v}{2^{j+1}}\right)-\frac{2^{\Delta J}}{q_{0}} \mathbb{E}\left[\prod_{\substack{j \in A \\
J_{1}<j \leq J_{2}}} h_{+}\left(\frac{\cdot}{2^{j+1-J_{1}}}\right)\right]\right|< \\
\kappa\left(q_{0}\right) \frac{2^{\Delta J}}{q_{0}} 2^{-\left|A \cap\left[J_{1}, J_{2}\right]\right|} .
\end{gathered}
$$

It will follow that

$$
\left|\sum_{q_{0} \mid x} f_{+}(x)-\frac{N}{q_{0}} \mathbb{E}\left[f_{+}\right]\right|<\kappa\left(q_{0}\right) 2^{-r} \frac{N}{q_{0}}
$$

(and similarly for $f_{-}$).

Denote

$$
\left.\left.A^{\prime}=A \cap\right] J_{1}, J_{2}\right]=J_{1}-1+\left\{j_{1}^{\prime}<\cdots<j_{r_{1}}^{\prime}\right\} \text { where } r_{1}<\frac{10 r \Delta J}{n} .
$$

Expanding as a Fourier series, we get

$$
\prod_{j \in A^{\prime}} h_{+}\left(\frac{v}{2^{j+1}}\right)-\mathbb{E}[\cdots]=\sum_{\left\{b_{j}\right\}_{j \in A^{\prime}}}^{*}\left[\prod_{j \in A^{\prime}} \widehat{h}_{+}\left(b_{j}\right)\right] e\left(\sum_{j \in A^{\prime}} \frac{b_{j}}{2^{j+1}} v\right)
$$

where $\sum^{*}$ refers to those $\left\{b_{j}\right\}_{j \in A^{\prime}}$ with $\sum_{j \in A^{\prime}} \frac{b_{j}}{2^{j+1-J_{1}}} \neq 0(\bmod 1)$.

Recall that $\left|b_{j}\right|<n^{3}$ and

$$
\sum_{\left\{b_{j}\right\}} \prod_{j \in A^{\prime}}\left|\widehat{h}_{+}\left(b_{j}\right)\right|<(C \log n)^{r_{1}} .
$$

Clearly, the left side of (4.5) is at most

$$
\frac{2^{\Delta J}}{q_{0}} \sum_{\left\{b_{j}\right\}}^{*} \prod_{j \in A^{\prime}}\left|\widehat{h}_{+}\left(b_{j}\right)\right|\left\{1_{\left[\left\|q_{0}\left(\sum_{j \in A^{\prime}} \frac{b_{j}}{2^{j+1-J_{1}}}\right)\right\|<\frac{n^{2} q_{0}}{2^{\Delta}}\right]}+2^{-n}\right\}
$$

and we may take in (4.5)

$$
\kappa\left(q_{0}\right)=2^{r_{1}} \sum_{\left\{b_{j}\right\}}^{*} \prod_{j \in A^{\prime}}\left|\widehat{h}_{+}\left(b_{j}\right)\right| \cdot 1_{\left[\left\|q_{0} \sum_{j \in A^{\prime}} \frac{b_{j}}{2^{j+1-J_{1}}}\right\|<\frac{n^{2} q_{0}}{2^{\Delta}}\right]} .
$$


Denote $\beta_{s}=b_{j_{s}^{\prime}}\left(1 \leq s \leq r_{1}\right)$. Fix $\left\{\beta_{s}\right\}$ such that

$$
\sum_{s=1}^{r_{1}} \frac{\beta_{s}}{2^{j_{s}^{\prime}}} \neq 0(\bmod 1)
$$

and estimate for given $Q<B$

$$
\sum_{\substack{q \sim Q \\ q \text { odd }}} 1_{\left[\left\|q\left(\sum_{s} \frac{\beta_{s}}{2^{j_{s}^{\prime}}}\right)\right\|<\frac{n^{2} Q}{2^{\Delta J}}\right]} .
$$

Since $q$ is odd, $q\left(\sum_{s} \frac{\beta_{s}}{2^{j_{s}^{\prime}}}\right)=\frac{b}{2^{j_{r}^{\prime}}} \neq 0(\bmod 1)$ and, if $(4.10) \neq 0$, necessarily $2^{-j_{r_{1}}^{\prime}}<n^{2} Q 2^{-\Delta J}, 2^{-\frac{n}{10 r} \stackrel{(4.2)}{<}} 2^{J_{2}-J_{1}-j_{r_{1}}^{\prime}}<n^{2} Q$, implying

$$
Q>2^{\frac{n}{11 r}}
$$

Let $(4.10)=\frac{Q}{K}, K \leq Q$. From the pigeon hole principle, there is some $1 \leq q_{1} \leq K$ and $0 \leq a_{1}<q_{1}$ with

$$
\left\|\sum \frac{\beta_{s}}{2^{j_{s}^{\prime}}}-\frac{a_{1}}{q_{1}}\right\|<\frac{n^{2} Q}{2^{\Delta J}}
$$

Note that $q\left|\sum \frac{\beta_{s}}{2^{j_{s}^{\prime}}}\right|<4 Q \frac{n^{3}}{2^{j_{1}^{\prime}}}$. If $2^{j_{1}^{\prime}}>8 B n^{3}$, it would follow that $\left\|q \cdot\left(\sum \frac{\beta_{s}}{2^{j_{s}^{\prime}}}\right)\right\|=$ $q\left|\sum \frac{\beta_{s}}{2^{j_{s}^{\prime}}}\right|<Q n^{2} 2^{-\Delta J}$ and $2^{-j_{r_{1}}^{\prime}}<2 n^{j} 2^{-\Delta J}$, contradicting (4.2).

Hence $2^{j_{1}^{\prime}} \leq 8 n^{3} B$.

Let $D \gg 1$ be another parameter with

$$
r_{1} D<\frac{1}{10} \Delta J
$$

Clearly there is some $1 \leq s_{0} \leq r_{1}$ such that

$$
j_{s_{0}}^{\prime}<j_{1}^{\prime}+r_{1} D
$$

and, if $s_{0}<r_{1}$,

$$
j_{s_{0}+1}^{\prime}-j_{s_{0}}^{\prime}>D
$$

Hence

$$
2^{j^{\prime}}<8 n^{3} B 2^{r_{1} D}
$$


By (4.12), (4.15)

$$
\left\|\sum_{s \leq s_{0}} \frac{\beta_{s}}{2^{j_{s}^{\prime}}}-\frac{a_{1}}{q_{1}}\right\|<\frac{n^{2} B}{2^{\Delta J}}+\frac{n^{3}}{2^{j_{s_{0}}^{\prime}+D}}<\frac{1}{2^{j_{s_{0}}^{\prime}+\frac{1}{2} D}}
$$

assuming

$$
D>10 \log n \text { and } \Delta J>10 \log B
$$

(4.17) implies that either $q_{1} \geq 2^{\frac{1}{2} D}$, hence

$$
K>2^{\frac{1}{2} D}
$$

or

$$
\sum_{s \leq s_{0}} \frac{\beta_{s}}{2^{j_{s}^{\prime}}}=\frac{a_{1}}{q_{1}} \quad(\bmod 1)
$$

Assuming (4.20), we deduce from (4.10), (4.12) that

$$
\left\|q q_{1}\left(\sum_{s>s_{0}} \frac{\beta_{s}}{2^{j_{s}^{\prime}}}\right)\right\|<\frac{n^{2} q_{1} Q}{2^{\Delta J}}
$$

and

$$
\left\|\sum_{s>s_{0}} \frac{\beta_{s}}{2^{j_{s}^{\prime}}}\right\|<\frac{n^{2} Q}{2^{\Delta J}}
$$

By (4.18) and the preceding, $\left\|q q_{1}\left(\sum_{s>s_{0}} \frac{\beta_{s}}{2^{j_{s}^{\prime}}}\right)\right\|=q q_{1}\left\|\sum_{s>s_{0}} \frac{\beta_{s}}{2^{j_{s}^{\prime}}}\right\|$ so that $\left\|\sum_{s>s_{0}} \frac{\beta_{s}}{2^{j_{s}^{\prime}}}\right\|<\frac{2 n^{2}}{2^{\Delta J}}$. Again using that $j_{r_{1}}^{\prime}<\Delta J-\frac{n}{10 r}$, it follows that $\sum_{s>s_{0}} \frac{\beta_{s}}{2^{j_{s}^{\prime}}}=$ $0(\bmod 1)$ and

$$
\sum_{s} \frac{\beta_{s}}{2^{j_{s}^{\prime}}}=\frac{a_{1}}{q_{1}} \neq 0(\bmod 1)
$$

recalling (4.9).

But then from (4.10), since $q_{1}$ is a power of 2 and $q$ is odd

$$
\frac{1}{q_{1}} \leq\left\|q \frac{a_{1}}{q_{1}}\right\|<\frac{n^{2} B}{2^{\Delta J}}
$$

(a contradiction).

Thus (4.19) holds and

$$
(4.10)<2^{-\frac{1}{2} D} Q
$$


Returning to (4.8), (4.7), (4.22) and the bound $r_{1}<\frac{10 r \Delta J}{n}$ imply that

$$
\sum_{\substack{q \sim Q \\ q \text { odd }}} \kappa(q)<(C \log n)^{\frac{10 r \Delta J}{n}} 2^{-\frac{1}{2} D} Q .
$$

Recalling (4.13), take $D=\frac{1}{200} \frac{n}{r}$. Taking $\Delta J \sim \log B$ and assuming

$$
\log B<c(\log \log n)^{-1} \frac{n^{2}}{r^{2}}
$$

we obtain

$$
(4.23)<2^{-10^{-3} \frac{n}{r}} Q
$$

Returning to (3.14), it follows in particular from the preceding that

$$
\begin{gathered}
N 2^{-r}(\log B)^{2} \sum_{\substack{q<B \\
q s f, \text { odd }}} \frac{\kappa(q)}{q}< \\
N 2^{-r}(\log B)^{2}\left(2^{-\frac{n}{12 r}} \cdot \log B+2^{-10^{-3} \frac{n}{r}} \log B\right)<N 2^{-r} 2^{-10^{-4} \frac{n}{r}}
\end{gathered}
$$

assuming (4.4), (4.24).

Going over our analysis, it is clear that in (4.5) the condition $v \equiv-u-$ $w\left(\bmod q_{0}\right)$ can be replaced by $v \equiv a\left(\bmod q_{0}\right)$ for arbitrary $a$. Also, $J_{1}$ may be taken at least $\frac{n}{2}$, so that the element $v \equiv 0\left(\bmod 2^{\left[\frac{n}{2}\right]}\right)$.

This variant of the above argument gives the following statement.

Let $q_{0}<B$ be odd and $m<\frac{n}{2}$. Then

$$
\sum_{\substack{x \equiv a_{0}\left(\bmod q_{0}\right) \\ x \equiv a^{\prime}\left(\bmod 2^{m}\right)}} f(x)=\frac{1}{q_{0}}\left[1+0\left(\kappa\left(q_{0}\right)+2^{-\frac{n}{12 r}}\right)\right]\left[\sum_{\substack{\left.x \equiv a^{\prime} \\ \bmod 2^{m}\right)}} f(x)\right] .
$$

From (4.27), we derive a character sum estimate relevant to (3.17).

Let $q_{1}=\tilde{q}_{1} 2^{m}\left(1<\tilde{q}_{1}\right.$ odd $), q_{1}<B$ and $\chi_{1}=\tilde{\chi}_{1} \chi^{\prime}$ with $\tilde{\chi}_{1}$ non-principal $\left(\bmod \tilde{q}_{1}\right)$ and $\chi^{\prime}\left(\bmod 2^{m}\right)$. Let $q_{3}<B$ be square free, odd and $\left(q_{1}, q_{3}\right)=1$. 
We apply (4.27) with $q_{0}=\tilde{q}_{1} q_{3}$. Clearly

$$
\begin{aligned}
\left|\sum_{q_{3} \mid x} \chi_{1}(x) f(x)\right| & \leq \sum_{a^{\prime}=1}^{2^{m}-1}\left|\sum_{a=1}^{\tilde{q}_{1}} \tilde{\chi}_{1}(a)\left\{\sum_{\substack{x \equiv a^{\prime}\left(\bmod 2^{m}\right) \\
x \equiv a\left(\bmod \tilde{q}_{1}\right) \\
q_{3} \mid x}} f(x)\right\}\right| \\
& \stackrel{(4.27)}{\lesssim} \frac{1}{q_{3}}\left(\kappa\left(q_{3} \tilde{q}_{1}\right)+2^{-\frac{n}{12 r}}\right) 2^{-r} N .
\end{aligned}
$$

Similarly, if $J \subset[1, N]$ is an interval of size $\frac{N}{B}$

$$
\left|\sum_{x \in J, q_{3} \mid x} \chi_{1}(x) f(x)\right| \lesssim \frac{1}{q_{3}}\left(\kappa\left(q_{3} \tilde{q}_{1}\right)+2^{-\frac{n}{12 r}}\right) 2^{-r}|J| .
$$

Hence, in (3.17)

$$
\alpha\left(q_{1}, q_{3}\right) \lesssim \kappa\left(q_{3} \tilde{q}_{1}\right)+2^{-\frac{n}{12 r}}
$$

Recall (4.23), (4.25), it follows that in (3.19)

$$
\begin{aligned}
\sum_{q_{3}<B} \frac{\alpha\left(q_{1}, q_{3}\right)}{q_{3}} & \lesssim(\log B) 2^{-\frac{n}{12 r}}+\sum_{q_{3}<B} \frac{\kappa\left(q_{3} \tilde{q}_{1}\right)}{q_{3}} \\
& \lesssim(\log B) 2^{-\frac{n}{12 r}}+\tilde{q}_{1} \sum_{q<B \tilde{q}_{1}} \frac{\kappa(q)}{q} \\
& \lesssim \tilde{q}_{1} 2^{-10^{-3} \frac{n}{r}} \log N
\end{aligned}
$$

which is conclusive for $1<\tilde{q}_{1}<2^{\frac{1}{2} 10^{-3} \frac{n}{r}}$.

\section{Further Estimates (II)}

Next, we establish a second bound on $\alpha\left(q_{1}, q_{0}\right)$.

Take $J=[1, N]$; the adaptation for $J$ an interval of size $\frac{N}{B}$ is straightforward.

Proceeding as in $\S 4$, take $J_{1}<J_{2}$ satisfying (4.2), (4.3) and $\Delta J=J_{2}=J_{1} \sim$ $\log B$. Write $x=\sum_{j<J_{1}} x_{j} 2^{j}+\sum_{J_{1} \leq j<J_{2}} x_{j} 2^{j}+\sum_{J_{2} \leq j<n} x_{j} 2^{j}=u+v+w$. 
Evaluate

$$
\left|\sum_{q_{0} \mid k} f(k) \mathcal{X}_{1}(k)\right| \leq \sum_{u, w}\left|\sum_{v \equiv-u-w} f(u+v+w) \mathcal{X}_{1}(u+v+w)\right|
$$

by evaluating the inner sum with $u, w$ fixed $\left(\equiv\right.$ refers to $\left.\left(\bmod q_{0}\right)\right)$.

Using the notations from $\S 4$

$$
\begin{gathered}
(5.1) \leq \sum_{u, w} \prod_{j \in A, j<J_{1}} h\left(\frac{u}{2^{j}}\right) \prod_{j \in A, j>J_{2}} h\left(\frac{w}{2^{j}}\right)\left|\sum_{v \equiv-u-w} \prod_{j \in A, J_{1}<j<J_{2}} h\left(\frac{v}{2^{j}}\right) \mathcal{X}_{1}(u+v+w)\right| \\
+2^{-\frac{n}{12 r}} \sum_{q_{0} \mid k} f_{+}(k) .
\end{gathered}
$$

It follows in particular from the analysis in $\S 4$ that

$$
(5.3)<2^{-\frac{n}{12 r}} 2^{-r} \frac{N}{q_{0}}
$$

Denoting $F(v)=\prod_{j \in A, J_{1}<j<J_{2}} h\left(\frac{v}{2^{j}}\right)$, we have (cf. (4.7))

$$
\begin{aligned}
\|\hat{F}\|_{1} & <(C \log n)^{\left|A \cap\left[J_{1}, J_{2}\right]\right|} \\
& <(C \log n)^{10 \frac{r}{n} \Delta J} \\
& <B^{c \frac{r}{n} \log \log n} .
\end{aligned}
$$

Recall that $\left(q_{0}, q_{1}\right)=1$ and $\mathcal{X}_{1}\left(\bmod q_{1}\right)$ is primitive.

Expanding $F$ in additive characters $\left(\bmod q_{1}\right)$ and applying the Gauss sum bound, it follows from the preceding that

$$
\left|\sum_{v \equiv-u-w} F(v) \mathcal{X}_{1}(u+v+w)\right| \ll\left(\log q_{1}\right) B^{C \frac{r}{n} \log \log n}\left(1+\frac{2^{\Delta J}}{q_{0} q_{1}}\right) \sqrt{q_{1}} .
$$

Hence

$$
\begin{aligned}
(5.2) & <2^{n-\Delta J-\left|A \backslash\left[J_{1}, J_{2}\right]\right|} \cdot(5.6) \\
& <\frac{N 2^{-r}}{q_{0}} C^{\frac{r}{n} \Delta J} B^{C \frac{r}{n} \log \log n}\left(q_{0} \sqrt{q_{1}} 2^{-\Delta J}+\frac{1}{\sqrt{q_{1}}}\right) \\
& <\frac{N 2^{-r}}{q_{0}}\left(\frac{1}{B}+\frac{1}{\sqrt{q_{1}}} B^{C \frac{4}{n} \log \log n}\right) \text { for appropriate } \Delta J \sim \log B
\end{aligned}
$$




$$
<\frac{N 2^{-r}}{q_{0}} \frac{1}{\sqrt{q_{1}}} B^{C \frac{r}{n} \log \log n}
$$

and it follows that

$$
\alpha\left(q_{1}, q_{0}\right)<2^{-\frac{n}{12 r}}+\frac{1}{\sqrt{q_{1}}} B^{C \frac{r}{n} \log \log n} .
$$

Hence in (3.19), there is also the estimate

$$
\sum_{q_{3}<B} \frac{\alpha\left(q_{1}, q_{3}\right)}{q_{3}}<2^{-\frac{n}{12 r}}(\log B)+\frac{B^{C \frac{r}{n} \log \log n}}{\sqrt{\tilde{q}_{1}}}(\log B) .
$$

Combined with (4.31), it follows that for $\tilde{q}_{1}>1$

$$
\sum_{q_{3}<B} \frac{\alpha\left(q_{1}, q_{3}\right)}{q_{3}}<2^{-\frac{1}{3} 10^{-3} \frac{n}{r}}
$$

provided

$$
\log B<c \frac{n^{2}}{r^{2}}(\log \log n)^{-1} .
$$

as already implied by (4.24).

\section{Conclusion of the Argument}

In this section, we address the subdivision in 'good' and 'bad' characters and complete the argument.

Denote $N(\alpha, T ; \chi)$ the number of zero's of $L(s, \chi)$ such that

$$
\alpha \leq \sigma \leq 1,|t| \leq T \quad(s=\sigma+i t)
$$

Then (see [Bom], Theorem 14)

$$
N(\alpha)=\sum_{q \leq Q} \sum_{\chi(\bmod q)}^{*} N(\alpha, T ; \chi) \ll(T Q)^{8(1-\alpha)}
$$

where $\sum^{*}$ refers to summation over primitive characters.

Let $\chi$ be a non-principal character. From Proposition 5.25 in [I-K], for $T \leq x$

$$
\psi(x, \chi)=-\sum_{\substack{L(\rho, \chi)=0 \\|\gamma| \leq T}} \frac{x^{\rho}-1}{\rho}+0\left(\frac{x}{T}(\log x q)^{2}\right)
$$


where $\rho=\beta+i \gamma$. We denote

$$
\eta=\eta(\chi)=\min (1-\beta)
$$

with min taken over all zero's $\rho$ of $L(s, \chi)$ with $|\gamma| \leq T$.

The subdivision of characters in classes $\mathcal{G}$ and $\mathcal{B}$ depends on whether $\eta \geq \eta^{*}$ (resp. $\eta<\eta^{*}$ ) with $\eta^{*}$ to be specified. Recall also that $q \leq B$.

It follows from (6.2) that

$$
|\psi(x, \chi)| \leq x\left(\sum_{\rho} \frac{1}{\rho x^{1-\beta}}+\frac{(\log x)^{2}}{T}\right)
$$

and from the density bound and (6.3), assuming $q T<x^{\frac{1}{20}}$

$$
\begin{aligned}
\sum \frac{1}{x^{1-\beta}} & =-2 \int_{\frac{1}{2}}^{1-\eta} \frac{1}{x^{1-\alpha}} d N(\alpha)=2 x^{-\frac{1}{2}} N\left(\frac{1}{2}\right)+2 \log x \int_{\eta}^{\frac{1}{2}}\left[\frac{(T Q)^{8}}{x}\right]^{\tau} d \tau \\
& <\frac{(q T)^{4}}{x^{1 / 2}}+x^{-\eta / 2}<x^{-\eta / 2} .
\end{aligned}
$$

Hence, for all $x$,

$$
|\psi(x, \chi)|<(Q T)^{20}+\frac{x(\log x)^{2}}{T}+x^{1-\frac{\eta}{2}}
$$

Fix $B<T=T(N)<N^{\frac{1}{100}}$. Assuming $\eta \geq \eta_{*}$, substitution of (6.5) in (2.7) gives $(r=|A|)$

$$
B(C \log n)^{r}\left\{(B T)^{20}+\frac{n^{2} N}{T}+N^{1-\frac{1}{2} \eta^{*}}\right\}
$$

If we let

$$
\eta_{*}=C \frac{\log T}{\log N}
$$

(with appropriate $C>0$ ), the contribution of

$$
\mathcal{G}=\left\{\chi(\bmod q) ; q<B, \eta(\chi) \geq \eta_{*}\right\}
$$

in (2.7) will be bounded by

$$
B(C \log n)^{r} n^{2} \frac{N}{T} .
$$


Next, consider the contribution of primitive $\chi_{1}\left(\bmod q_{1}\right)$ in $\mathcal{B}$, given by (3.19).

Assuming $\tilde{q}_{1}>1$, (5.11) implies

$$
2^{-r} N(\log N)^{5} \sum_{\chi_{1} \in \mathcal{B}} \frac{q_{1}}{\phi\left(q_{1}\right)} \sum_{\substack{q_{3}<B \\ q_{3} s f, \text { odd }}} \frac{\alpha\left(q_{1}, q_{3}\right)}{q_{3}}<2^{-r} N n^{6} 2^{-\frac{1}{3} 10^{-3} \frac{n}{r}}|\mathcal{B}|
$$

where again by $(6.1)$

$$
|\mathcal{B}|=\mid\left\{\chi_{1} \text { primitive }\left(\bmod q_{1}\right) \text { with } q_{1}<B \text { and } \eta\left(\chi_{1}\right)<\eta_{*}\right\} \mid<(T B)^{8 \eta_{*}} \text {. }
$$

Hence

$$
(6.9)<2^{-r} N 2^{-\frac{1}{4} 10^{-3} \frac{n}{r}}
$$

provided

$$
\log T<c \frac{n}{\sqrt{r}}
$$

(for appropriate $c>0$ ).

Recall also assumption (4.24)

$$
\log B<c(\log \log n)^{-1} \frac{n^{2}}{r^{2}}
$$

Finally, consider the case $\tilde{q}_{1}=1$, i.e. $q_{1}$ is a power of 2 .

From the Gallagher-Iwaniec result (cf. [H-K], Lemma 5), we obtain the following improved zero-free region

$$
\eta\left(\chi_{1}\right) \gtrsim\left[\left(\log q_{1} T\right)\left(\log \log q_{1} T\right)\right]^{-3 / 4} \gg(\log T \cdot \log \log T)^{-3 / 4} .
$$

In order for $\chi$ to be induced by such $\chi_{1}$ to be in $\mathcal{G}$, we require

$$
\frac{\log T}{\log N}<c(\log T \cdot \log \log T)^{-3 / 4}
$$

i.e

$$
\log T \ll n^{4 / 7}(\log n)^{-3 / 7} .
$$

Collecting the bounds (1.11), (6.8), (3.15), (4.26), (3.19), (6.11), we obtain

$$
(1.1)-2 \mathbb{E}[f] N=
$$


$N 2^{-r} O\left\{\frac{n^{3}(C \log n)^{r}}{\sqrt{B}}+\frac{n^{2} B(C \log n)^{r}}{T}+B 2^{r} \exp \left(-c \frac{n^{3 / 5}}{(\log n)^{1 / 5}}\right)+2^{-10^{-4} \frac{n}{r}}\right\}$.

Fix $0<\gamma<1$. If we let

$$
B=n^{6}(C \log n)^{2 r} \gamma^{-2} \text { and } T=n^{8}(C \log n)^{3 r} \gamma^{-3}
$$

and in order to fulfill (6.12), (6.13), (6.15), we assume

$$
r<\left(\frac{n}{\log n}\right)^{4 / 7}
$$

then (6.16) implies

$$
(1.1)-2 \mathbb{E}[f] N<\gamma \mathbb{E}[f]
$$

This proves the following

Theorem. Let $N=2^{n}$, $n$ large enough. Under assumption (6.17), choosing a set $A \subset\{1, \ldots, n\},|A|=r$ of binary digits and assigned values $\alpha_{j}=0,1$ for $j \in A$, we have

$$
\begin{gathered}
\mid\left\{p<N ; p \text { has } j \text {-digit equal to } \alpha_{j} \text { for } j \in A\right\} \mid= \\
(1+o(1)) \frac{N}{\log N} 2^{-r} .
\end{gathered}
$$

The argument permits to give a better error term that we do not specify here. Acknowledgement. The author is grateful to the referee for his comments and suggestions to improve the presentation.

\section{REFERENCES}

[Bom] E. Bombieri, Le grand crible dans la théorie analytique des nombres, Astérisque 18.

[D] H. Davenport, Multiplicative number theory, Springer GTM 74.

[Ga] P.X. Gallagher, Primes in progressions to prime-power modulus, Inventiones 16 (1972), 191-201.

[I] H. Iwaniec, On zero's of Dirichlet L-series, Inventiones 23 (1974), 97-104.

[I-K] H. Iwaniec, E. Kowalski, Analytic number theory, AMS Colloquium Publ, Vol. 53. 
[H-K] G. Harman, I. Katai, Primes with preassigned digits II, Acta. Arith. 133.2 (2008), 171-184.

School of Mathematics, Institute for Advanced Study, Princeton, NJ 08540 